\title{
The ratio of contrast volume to glomerular filtration rate predicts in-hospital and six-month mortality in patients undergoing primary angioplasty for ST-elevation myocardial infarction
}

\author{
Gökhan Çiçek ${ }^{1}$, Mehmet Bozbay², Sadık Kadri Açıkgoz ${ }^{3}$, Servet Altay ${ }^{4}$, \\ Murat Uğur ${ }^{5}$, Bayram Köroğlu ${ }^{5}$, Huseyin Uyarel $^{6}$
}

${ }^{1}$ Department of Cardiology, Ankara Numune Education and Research Hospital, Ankara, Turkey ${ }^{2}$ Department of Cardiology, Marmara University Pendik Research and Training Hospital, Istanbul, Turkey ${ }^{3}$ Department of Cardiology, Turkiye Yuksek Ihtisas Training and Research Hospital, Ankara, Turkey

${ }^{4}$ Department of Cardiology, Edirne Devlet Hastanesi, Edirne, Turkey

${ }^{5}$ Department of Cardiology, Siyami Ersek Center for Cardiovascular Surgery, Istanbul, Turkey ${ }^{6}$ Department of Cardiology, Faculty of Medicine, Bezmi Alem Vakıf University, Istanbul, Turkey

\section{Abstract}

Background: The aim of this study is to determine the impact of ratio of contrast volume to glomerular filtration rate (V/GFR) on development of contrast-induced nephropathy (CIN) and long-term mortality in patients with ST-segment elevation acute myocardial infarction (STEMI) undergoing primary percutaneous coronary intervention (PCI).

Methods: A total of 645 patients with STEMI undergoing primary PCI was prospectively enrolled. CIN was defined as an absolute increase in serum creatinine $>0.5 \mathrm{mg} / \mathrm{dL}$ or a relative increase $>25 \%$ within $48 \mathrm{~h}$ after PCI. The study population was divided into tertiles based on V/GFR. A high V/GFR was defined as a value in the third tertile (> 3.7).

Results: Patients in tertile 3 were older, had higher rate of smoking, diabetes mellitus and CIN, lower left ventricular ejection fraction, hemoglobin, and systolic and diastolic blood pressure compared to tertiles 1 and $2(p<0.05)$. V/GFR was found an independent predictor of in-hospital and 6-month mortality. We found 2 separate values of V/GFR for 2 different end points. While the ratio of 3.6 predicted in-hospital mortality with $78 \%$ sensitivity and $82 \%$ specificity, the ratio of 3.3 predicted 6 -month mortality with $71 \%$ sensitivity and $76 \%$ specificity. Survival rate decreases as V/GFR increases both for in-hospital and during 6-month follow-up. Diabetes mellitus and multivessel disease were other predictors of in-hospital mortality. Conclusions: High V/GFR level is associated with increased in-hospital and long-term mortality in patients with STEMI undergoing primary PCI. (Cardiol J 2015; 22, 1: 101-107)

Key words: contrast-induced nephropathy, contrast volume to glomerular filtration rate ratio, ST-segment elevation myocardial infarction

Address for correspondence: Gökhan Çiçek, MD, Talatpasa Bulvari, 06100, Sihhiye, Ankara, Turkey, tel: +90 31250840 00, fax: +90 31231034 60, e-mail: drvaristor@hotmail.com 


\section{Introduction}

Decreased glomerular filtration rate (GFR) is an important predictor of adverse cardiovascular events in acute coronary syndromes [1-5]. Patients with normal renal functions who undergo percutaneous coronary interventions (PCI) are also at risk of adverse events due to contrast-induced nephropathy (CIN) [6, 7].

Contrast volume is a modifiable major risk factor for CIN and closely related with in-hospital mortality [8-10]. Recently, the ratio of contrast volume to GFR (V/GFR) was found a predictor of increase in creatinine values and CIN following PCI [11-13]. However, the impact of V/GFR on short- and long-term prognosis after primary PCI has not been evaluated so far. Therefore, we aimed to investigate the impact of V/GFR for predicting adverse cardiac events after primary PCI for ST-segment elevation acute myocardial infarction (STEMI) [14].

\section{Methods}

\section{Study population}

We prospectively enrolled 645 consecutive patients with STEMI who were admitted to the Emergency Department and underwent urgent cardiac catheterization procedures between December 2009 and July 2011. The patients were assigned into tertiles according to their V/GFR. The patients were enrolled into the study if they fulfilled the following criteria: (i) the onset of symptoms presenting within $12 \mathrm{~h}$ (typical chest pain lasting for $>30 \mathrm{~min}$ ); (ii) $\geq 1 \mathrm{~mm}$ ST-segment elevation in at least 2 contiguous electrocardiogram (ECG) leads except $V_{2}-V_{3}$ which required $1.5 \mathrm{~mm}$ for female and $2 \mathrm{~mm}$ for male patients or new onset left bundle-branch block; (iii) treatment with primary PCI (angioplasty and/or stent deployment). All primary PCI procedures were performed in a single high-volume tertiary center $(>3,000 \mathrm{PCI} /$ /year) by expert operators performing more than 75 PCIs per year (15 of them are primary PCI). The study protocol was approved by the Institutional Ethics Committee.

\section{Analysis of patient data}

The cardiovascular history, demographic information, and risk factors (diabetes mellitus, smoking, hypertension and hypercholesterolemia) of the patients were obtained from their medical records. Reperfusion time and door-to-balloon time were also recorded. On admission, blood values were obtained from all patients. Serum creatinine was also measured before the angiography procedure and within $48 \mathrm{~h}$ afterwards. A 12-lead ECG was recorded in each patient just after hospital admission, and the myocardial infarction type was also obtained from the ECGs. Twenty four to $72 \mathrm{~h}$ after revascularization, a transthoracic echocardiography was performed by using system $\mathrm{V}$ (Vingmed, GE, Horten, Norway) with a $2.5-\mathrm{MHz}$ phased-array transducer, and the left ventricular ejection fraction (LVEF) was calculated using a modified Simpson's method [15].

\section{Coronary angiography and PCI}

All patients were given a chewable $300 \mathrm{mg}$ aspirin and $600 \mathrm{mg}$ loading dose of clopidogrel before coronary angiography. After the procedure, all patients were prescribed $100 \mathrm{mg}$ of aspirin and $75 \mathrm{mg}$ of clopidogrel daily. Angiographic data of the patients were assessed from the catheter laboratory records. All procedures were performed via femoral route. A nonionic, low osmolar contrast agent (Iopromide, $370 \mathrm{mg} / \mathrm{mL}$ Bayer HealthCare Pharmaceuticals Inc., Germany) was utilized in all patients. The artery that was anticipated to be unhindered was injected first. Heparin $(100 \mathrm{IU} / \mathrm{kg}$ ) was administered when the coronary anatomy was first described. After visualizing the left and right coronary arteries, $200 \mu \mathrm{g}$ of nitroglycerine was selectively injected into the infarct related artery (IRA) to exclude a possible coronary spasm. Angiographic evaluation was made by visual assessment. Primary angioplasty (including balloon angioplasty and/or stent implantation) was performed just for IRA according to lesion type. For each procedure, interventional success at the acute phase was defined as reducing to $<30 \%$ of obstruction and stenosis of the IRA with Thrombolysis in Myocardial Infarction 3 flow just after primary angioplasty. The use of tirofiban was left to the discretion of the operator.

\section{Definitions}

The time from symptom onset to the coronary reperfusion was defined as time to reperfusion with balloon inflation. Door-to-balloon time was defined as the time between Emergency Department and balloon inflation. Patients were assessed according to Killip clinical examination classification [16]. Advanced heart failure was defined as New York Heart Association (NYHA) class 3 and 4. Non-diabetic patients were defined as the patients without documented diabetes using neither oral hypoglycemic agents nor insulin treatment at 
admission. Hypercholesterolemia was defined as total cholesterol of at least $200 \mathrm{mg} / \mathrm{dL}$ or use of cholesterol-lowering drugs. A family history of coronary artery disease (CAD) was defined as a documented case of $\mathrm{CAD}$ in a parent or sibling before 60 years of age. Anemia was defined as hemoglobin concentration lower than $13 \mathrm{mg} / \mathrm{dL}$ in men and $12 \mathrm{mg} / \mathrm{dL}$ in women. We defined repeat target vessel revascularization as a necessity for PCI or coronary surgery due to restenosis or reocclusion of the IRA. Reinfarction was defined as an elevation of serum creatinine kinase myocardial band level more than 2 times from the upper limit of normal and ST-segment re-elevation.

The estimated GFR (eGFR) was calculated by using the modified Modification of Diet in Renal Disease (MDRD) equation [17]: eGFR (mL/ $\left./ \mathrm{min} / 1.73 \mathrm{~m}^{2}\right): 186 \times(\mathrm{SCr})^{-1.154} \times(\text { age })^{-0.203}(\times 0.742$ if women), where $\mathrm{SCr}$ defines serum creatinine concentration in milligrams per deciliter as measured immediately before PCI, and age is given in years. The V/GFR ratio was calculated by dividing the volume of contrast medium used during the PCI by the patient's eGFR. CIN was defined as an increase in SCr by either $>0.5 \mathrm{mg} / \mathrm{dL}$ or by $>25 \%$ from baseline within the first $2-3$ days after contrast medium administration, when another explanations for renal insufficiency have been excluded [18].

\section{Statistical analysis}

Analyses were performed using SPSS Statistics, version 17.0 (SPSS Inc, Chicago, IL). To test the distribution pattern, the Kolmogorov-Smirnov method was used. Data were summarized as a mean standard deviation, median and interquartile range, or proportions. The Student's t-test was used to compare data with normal distribution and the Mann-Whitney U test was applied to compare the data without normal distribution. Categorical variables were compared with the $\chi^{2}$ test. The effects of different variables on clinical outcomes were calculated by univariate analysis for each. The variables for which the unadjusted $\mathrm{p}$ value was $<0.10$ in Cox regression analysis were identified as potential risk markers and included in the multivariable Cox regression model. An exploratory evaluation for additional cut points of different variables was performed using the receiver operating characteristic (ROC) curve analysis. The survival curve during hospitalization for V/GFR was analyzed using the Kaplan-Meier method, and statistical assessment was performed using the log-rank test. A p value $<0.05$ was considered statistically significant for all analyses.

\section{Results}

Baseline characteristics of the study population are shown in Table 1. Patients in tertile 3 were older, had higher rate of smoking, diabetes mellitus and CIN, lower LVEF, hemoglobin, and systolic and diastolic blood pressure compared to tertiles 1 and $2(\mathrm{p}<0.05)$.

Nineteen patients died during hospital stay and 34 patients died within the first 6 months of the follow-up period. Univariable and multivariable Cox regression analyses revealed CIN (HR 2.99, confidence interval [CI] 1.96-4.02, $\mathrm{p}<0.001)$ and V/GFR (HR 1.07, CI 1.04-1.12, p < 0.001) as the predictors of in-hospital mortality (Table 2). Diabetes mellitus and multi-vessel disease were the other predictors of in-hospital mortality. CIN (HR 2.17, CI 1.47-2.84, $\mathrm{p}<0.001$ ) and V/GFR (HR 1.08, CI 1.02-1.15, p < 0.003) also predicted 6 -month mortality (Table 3). Apart from these findings, presence of diabetes mellitus, multi-vessel disease, and admission Killip class more than 1 independently predicted 6 -month mortality.

A ROC curve analysis revealed V/GFR ratio of 3.6 for prediction of in-hospital mortality with $78 \%$ sensitivity and $82 \%$ specificity $(\mathrm{C}$ statics $=$ $=0.850, \mathrm{p}<0.001)$. The V/GFR ratio of 3.3 predicted 6 -month mortality with $71 \%$ sensitivity and $76 \%$ specificity $(\mathrm{C}$ statics $=0.790, \mathrm{p}<0.001)$. Figure 1 shows Kaplan-Meier curves among tertiles for both in-hospital and 6-month mortality which represent worse outcome as the V/GFR increases.

\section{Discussion}

Our study results showed that V/GFR ratio independently predicted both in-hospital and 6 -month mortality in patients undergoing primary PCI. To our knowledge, our study is an original one in terms of evaluating the impact of V/GFR ratio on long-term mortality in STEMI patients undergoing primary PCI.

Patients who present with acute myocardial infarction and decreased GFR have higher mortality rate even after optimal treatment [19]. Presence of calcified atherosclerosis,-large vessel remodeling, left ventricular hypertrophy, chronic volume overload, and pressure overload in renal failure was thought to play a role in this situation. Additionally, comorbidities accompanying impaired GFR could explain this higher mortality after acute myocardial infarction [20-22]. Furthermore, cardiovascular medications and invasive therapeutic strategies are underused in patients with poor 
Table 1. Clinical and hematologic characteristics of ratio of contrast volume to glomerular filtration rate (V/GFR) tertiles.

\begin{tabular}{|c|c|c|c|c|}
\hline \multirow[t]{2}{*}{ Variables } & \multicolumn{3}{|c|}{ V/GFR } & \multirow[t]{2}{*}{$\mathbf{P}$} \\
\hline & $\begin{array}{c}\text { Tertile } 1 \\
\text { (n = 215) } \\
1.34(1.1-1.6)\end{array}$ & $\begin{array}{c}\text { Tertile } 2 \\
(n=215) \\
2.1(1.9-2.4)\end{array}$ & $\begin{array}{c}\text { Tertile } 3 \\
(n=215) \\
3.7(3.2-4.8)\end{array}$ & \\
\hline Age [years] & $50.0 \pm 10.1$ & $56.6 \pm 11.4$ & $62.8 \pm 11.8$ & $<0.001$ \\
\hline Male & $200(93 \%)$ & $183(85 \%)$ & $167(77 \%)$ & $<0.001$ \\
\hline Female & $15(7 \%)$ & $32(15 \%)$ & $48(229 \%)$ & \\
\hline Diabetes mellitus & $32(15 \%)$ & $39(18 \%)$ & $55(26 \%)$ & 0.016 \\
\hline Hypertension & $79(37 \%)$ & $90(42 \%)$ & $96(45 \%)$ & 0.240 \\
\hline Peripheral arterial disease & $6(3 \%)$ & $4(2 \%)$ & $7(3 \%)$ & 0.655 \\
\hline Post TIMI 1 & $4(2 \%)$ & $3(1 \%)$ & $10(5 \%)$ & \\
\hline Post TIMI 2 & $9(4 \%)$ & $11(5 \%)$ & $10(5 \%)$ & 0.097 \\
\hline Post TIMI 3 & $202(94 \%)$ & $201(94 \%)$ & $195(91 \%)$ & \\
\hline Contrast-induced nephropathy & $17(8 \%)$ & $23(11 \%)$ & $48(22 \%)$ & $<0.001$ \\
\hline Ejection fraction [\%] & $45(40-50)$ & $45(40-50)$ & $45(40-50)$ & 0.004 \\
\hline Glucose [mg/dL] & $131(112 / 169)$ & $137(113 / 166)$ & $147(119 / 196)$ & 0.007 \\
\hline $\mathrm{HDL}[\mathrm{mg} / \mathrm{dL}]$ & $37(31-43)$ & $38(31-43)$ & $38(32-48)$ & 0.147 \\
\hline LDL [mg/dL] & $124.8 \pm 36.6$ & $122.7 \pm 36.4$ & $111.8 \pm 37.6$ & $<0.001$ \\
\hline Triglycerides [mg/dL] & $148(100 / 199)$ & $142(95 / 205)$ & $126(90 / 176)$ & 0.022 \\
\hline Hemoglobin [g/L] & $14.6(13.6 / 15.5)$ & $14.1(13.1 / 15.1)$ & $13.5(12.5 / 14.9)$ & $<0.001$ \\
\hline Total WBC $\left[\times 10^{9} / L\right]$ & $12.3(10.3 / 14.6)$ & $12(10.1 / 14)$ & $11.9(10 / 14.6)$ & 0.561 \\
\hline Peak CK-MB [U/L] & $141(75-216)$ & $123(69-211)$ & $149(81-271)$ & 0.098 \\
\hline Early creatinine [mg/dL] & $0.8(0.7-0.9)$ & $0.9(0.8-1)$ & $1(0.85-1.2)$ & $<0.001$ \\
\hline Creatinine clearance $[\mathrm{mL} / \mathrm{min}]$ & $136(122-166)$ & 106 (89-129) & $76(61-100)$ & $<0.001$ \\
\hline Contrast volume [mL] & $200(100-200)$ & $200(200-300)$ & $300(200-400)$ & $<0.001$ \\
\hline Killip class $>1$ & $5(2.3 \%)$ & $7(3.3 \%)$ & $29(13.5 \%)$ & $<0.001$ \\
\hline Heart rate [bpm] & $80(72-84)$ & $80(70-85)$ & $80(70-85)$ & 0.835 \\
\hline In-hospital mortality & $0(0 \%)$ & $4(2 \%)$ & $15(7 \%)$ & $<0.001$ \\
\hline Six-month mortality & $2(1 \%)$ & $8(4 \%)$ & $24(11 \%)$ & $<0.001$ \\
\hline
\end{tabular}

TIMI - Thrombolysis in Myocardial Infarction; HDL — high density lipoprotein; LDL — low density lipoprotein; WBC — white blood cell; CK-MB - creatinine kinase myocardial band

Table 2. Significant predictors of in-hospital mortality in univariable and multivariable Cox regression analyses.

\begin{tabular}{|c|c|c|c|c|}
\hline \multirow[t]{2}{*}{ Variables } & \multicolumn{2}{|c|}{ Univariable } & \multicolumn{2}{|c|}{ Multivariable } \\
\hline & HR (95\% Cl) & $\mathbf{P}$ & $\operatorname{HR}(95 \% \mathrm{CI})$ & $\mathbf{P}$ \\
\hline Age & $1.05(1.02-1.07)$ & $<0.001$ & $1.03(0.95-1.13)$ & 0.245 \\
\hline Male/female & $1.02(0.80-1.19)$ & 0.189 & - & - \\
\hline Diabetes mellitus & $2.92(1.97-5.89)$ & $<0.001$ & $2.01(1.39-2.64)$ & $<0.001$ \\
\hline Smoking & $1.18(1.11-1.25)$ & 0.001 & $0.88(0.75-1.00)$ & 0.122 \\
\hline Killip class $>1$ & $15.2(6.65-21.7)$ & $<0.001$ & $7.71(3.78-23.9)$ & $<0.001$ \\
\hline Ejection fraction & $1.51(0.40-4.66)$ & $<0.001$ & $2.91(1.26-6.72)$ & 0.012 \\
\hline Contrast induced nephropathy & $4.05(2.02-6.27)$ & $<0.001$ & $2.99(1.96-4.02)$ & $<0.001$ \\
\hline V/GFR & $1.08(1.03-1.13)$ & $<0.001$ & $1.07(1.04-1.12)$ & 0.001 \\
\hline Low density lipoprotein & $1.14(0.88-1.41)$ & 0.622 & - & - \\
\hline Multi vessel disease & $2.51(1.53-3.04)$ & $<0.001$ & $2.55(1.74-3.34)$ & $<0.001$ \\
\hline Hemoglobin & $1.22(1.10-1.35)$ & 0.003 & $1.17(1.08-1.26)$ & 0.007 \\
\hline
\end{tabular}

$\mathrm{HR}$ - hazard ratio; $\mathrm{Cl}$ - confidence interval; V/GFR — contrast volume-glomerular filtration rate ratio 
Table 3. Significant predictors of mortality at 6-month follow-up in univariable and multivariable Cox regression analyses.

\begin{tabular}{|c|c|c|c|c|}
\hline \multirow[t]{2}{*}{ Variables } & \multicolumn{2}{|c|}{ Univariable } & \multicolumn{2}{|c|}{ Multivariable } \\
\hline & HR (95\% Cl) & $\mathbf{P}$ & HR (95\% CI) & $\mathbf{P}$ \\
\hline Age & $1.09(1.02-1.16)$ & 0.002 & $1.00(0.94-1.07)$ & 0.344 \\
\hline Male/female & $0.88(0.77-1.02)$ & 0.207 & - & - \\
\hline Diabetes mellitus & $3.01(1.88-4.17)$ & $<0.001$ & $2.61(1.89-3.23)$ & $<0.001$ \\
\hline Smoking & $1.04(0.94-1.15)$ & 0.411 & - & - \\
\hline Killip class $>1$ & $10.4(5.71-16.4)$ & $<0.001$ & $4.64(3.27-5.18)$ & $<0.001$ \\
\hline Ejection fraction & $1.04(1.02-1.06)$ & $<0.001$ & $1.02(1.01-1.03)$ & 0.009 \\
\hline Contrast induced nephropathy & $3.84(2.47-5.22)$ & $<0.001$ & $2.17(1.47-2.84)$ & $<0.001$ \\
\hline V/GFR & $1.06(1.01-1.12)$ & $<0.001$ & $1.08(1.02-1.15)$ & 0.003 \\
\hline Low density lipoprotein & $1.04(0.84-1.26)$ & 0.577 & - & - \\
\hline Multi vessel disease & $2.88(1.85-3.92)$ & $<0.001$ & $2.61(1.92-3.53)$ & $<0.001$ \\
\hline Hemoglobin & $1.27(1.13-1.43)$ & 0.001 & $1.22(1.10-1.36)$ & 0.024 \\
\hline
\end{tabular}

$\mathrm{HR}$ - hazard ratio; $\mathrm{Cl}$ - confidence interval; V/GFR — contrast volume-glomerular filtration rate ratio
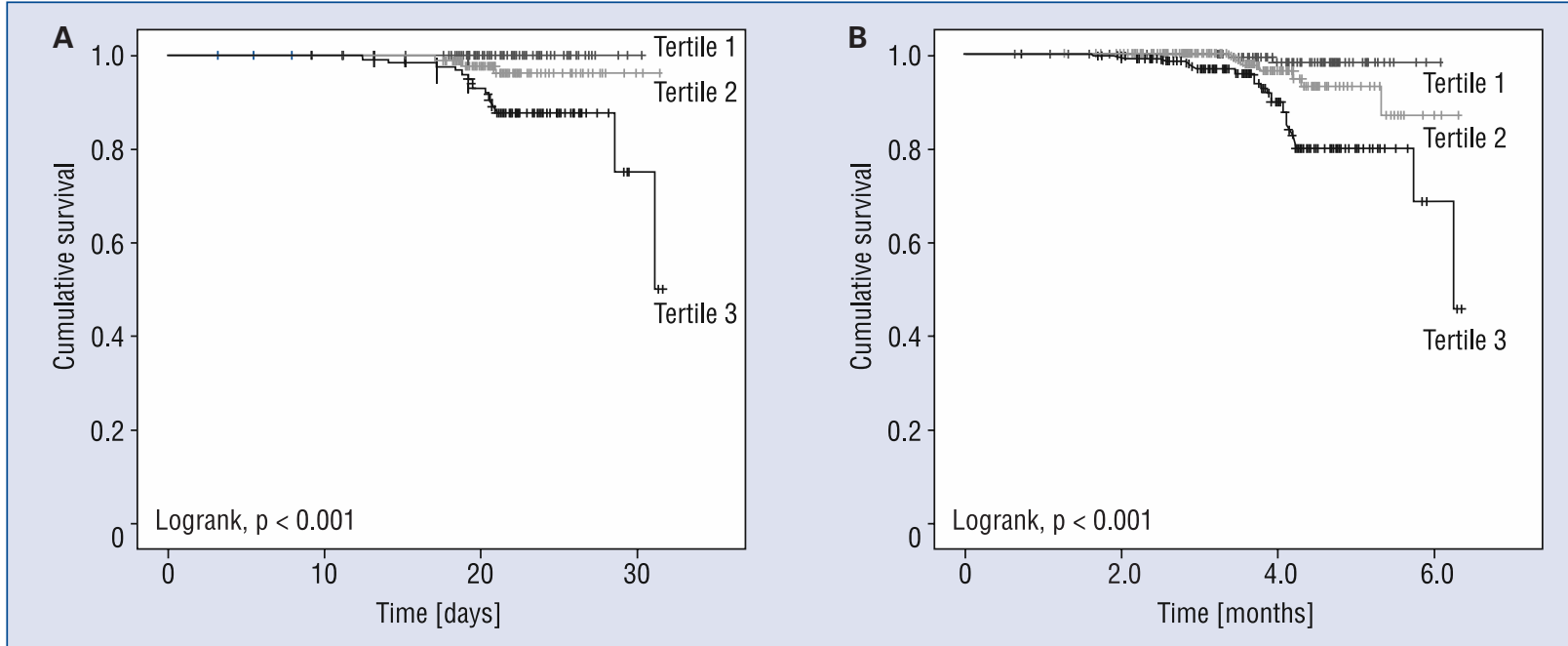

Figure 1. Kaplan-Meier curves of ratio of contrast volume to glomerular filtration rate (V/GFR) tertiles for in-hospital [A] and 6-month mortality [B].

renal function [4, 22-24]. However, presence of normal renal function may not guarantee favorable outcomes in terms of CIN and renal deterioration following coronary angiography is still possible. The kidneys are vulnerable to contrast-induced toxicity during primary PCI, but the mechanism and role of contrast agents in the pathogenesis of acute renal damage remain controversial and are not completely understood. Direct contrast-related toxicity and renal ischemia may play an important role due to circulatory impairment. An increase in serum creatinine during the acute phase of STEMI may be an indicator for more severe and extensive atherosclerosis and circulatory instability. Impaired renal function and radiographic contrast volume are important risk factors for development of CIN. Above all, the risk of CIN augments as the GFR values decrease [25]. As a major clinical determinant, the development of CIN is strongly associated with increased morbidity and mortality [26]. In 1 study, in-hospital mortality was found at a level of $22 \%$ among patients who developed CIN after PCI [25]. In another one, McCullough et al. [27] found an in-hospital mortality of $7 \%$ in patients who developed 
CIN after PCI and did not need hemodialysis, and $36 \%$ among those who needed hemodialysis after PCI. The contrast volume is a modifiable factor for the development of CIN. In patients presented with STEMI who underwent primary PCI, in-hospital mortality and CIN are closely associated with the contrast volume [6].

The ratio of contrast volume to GFR was proposed as a predictor of early increase in serum creatinine undergoing PCI. The ratio of 3.7 for V/GFR was found the cut-off value for predicting this increase [13]. Mager et al. [14] studied the association between V/GFR, post-procedural CIN and 1-month mortality in patients with STEMI. In line with Laskey et al. [13], the ratio of $>3.7$ was found to be associated with CIN and 1-month mortality.

In our study, V/GFR ratio was found an independent predictor of both in-hospital and 6-month mortality. We found 2 separate values of V/GFR for 2 different end points. The ratio of 3.6 predicted in-hospital mortality with $78 \%$ sensitivity and $82 \%$ specificity. On the other hand, the ratio of 3.3 predicted 6 -month mortality with $71 \%$ sensitivity and $76 \%$ specificity. Our values are different from those of the previous studies regarding the cut off V/GFR ratios. As in line with previous studies, V/GFR was associated with worse outcomes. Among tertiles, tertile 3 had a worse survival rate. Survival rates decrease as V/GFR increases both for in-hospital and during 6 -month follow-up periods.

\section{Conclusions}

V/GFR is an important parameter for predicting adverse events in STEMI patients undergoing primary PCI. Patients who had high V/GFR ratio should be followed closely to avert CIN especially in older patients with lower GFR. The impact of close follow-up on mortality and adverse events in high-risk population is unknown and needs further studies.

\section{Conflict of interest: None declared}

\section{References}

1. Best PJ, Lennon R, Ting HH, Bell MR, Rihal CS, Holmes DR, Berger PB. The impact of renal insufficiency on clinical outcomes in patients undergoing percutaneous coronary interventions. J Am Coll Cardiol, 2002; 39: 1113-1119.

2. AlSuwaidiJ, Reddan DN, Williams Ketal.; GUSTO-IIb, GUSTO-III, PURSUIT. Global Use of Strategies to Open Occluded Coronary Arteries. Platelet Glycoprotein IIb/IIIa in Unstable Angina: Receptor Suppression Using Integrilin Therapy; PARAGON-A Investigators. Platelet IIb/IIIa Antagonism for the Reduction of Acute coronary syndrome events in a Global Organization Ne- twork. Prognostic implications of abnormalities in renal function in patients with acute coronary syndromes. Circulation, 2002; 106: 974-980.

3. Gibson CM, Pinto DS, Murphy SA et al.; TIMI Study Group. Association of creatinine and creatinine clearance on presentation in acute myocardial infarction with subsequent mortality. J Am Coll Cardiol, 2003; 42: 1535-1543.

4. Normand ST, Glickman ME, Sharma RG, McNeil BJ. Using admission characteristics to predict short-term mortality from myocardial infarction in elderly patients. Results from the Cooperative Cardiovascular Project. JAMA, 1996; 275: 1322-1328.

5. Eagle KA, Lim MJ, Dabbous OH et al.; GRACE Investigators. A validated prediction model for all forms of acute coronary syndrome: Estimating the risk of 6 -month postdischarge death in an international registry. JAMA, 2004; 291: 2727-2733.

6. Marenzi G, Assanelli E, Campodonico J et al. Contrast volume during primary percutaneous coronary intervention and subsequent contrast-induced nephropathy and mortality. Ann Intern Med, 2009; 150: 170-177.

7. Rihal CS, Textor SC, Grill DE et al. Incidence and prognostic importance of acute renal failure after percutaneous coronary interventio. Circulation, 2002; 105: 2259-2264.

8. Marenzi G, Lauri G, Assanelli E et al. Contrast-induced nephropathy in patients undergoing primary angioplasty for acute myocardial infarction. Am Coll Cardiol, 2004; 44: 1780-1785.

9. Sadeghi HM, Stone GW, Grines CL et al. Impact of renal insufficiency in patients undergoing primary angioplasty for acute myocardial infarction. Circulation, 2003; 108: 2769-2775.

10. Marenzi G, Assanelli E, Marana I et al. N-acetylcysteine and contrast-induced nephropathy in primary angioplasty. N Engl J Med, 2006; 354: 2773-2782.

11. Mehran R, Aymong ED, Nikolsky E et al. A simple risk score for prediction of contrast-induced nephropathy after percutaneous coronary intervention: Development and initial validation. J Am Coll Cardiol, 2004; 44: 1393-1399.

12. Nyman U, Almén T, Aspelin P, Hellström M, Kristiansson M, Sterner G. Contrast-medium-Induced nephropathy correlated to the ratio between dose in gram iodine and estimated GFR in $\mathrm{ml} /$ min. Acta Radiol, 2005; 46: 830-842.

13. Laskey WK, Jenkins C, Selzer F et al.; NHLBI Dynamic Registry Investigators. Volume-to-creatinine clearance ratio: a pharmacokinetically based risk factor for prediction of early creatinine increase after percutaneous coronary intervention. J Am Coll Cardiol, 2007; 50: 584-590.

14. Mager A, Vaknin Assa H, Lev EI, Bental T, Assali A, Kornowski R. The ratio of contrast volume to glomerular filtration rate predicts outcomes after percutaneous coronary intervention for ST-segment elevation acute myocardial infarction. Catheter Cardiovasc Interv, 2011; 78: 198-201.

15. Schiller NB, Shah PM, Crawford M et al. Recommendations for quantitation of the left ventricle by two-dimensional echocardiography. American Society of Echocardiography Committee on Standards, Subcommittee on Quantitation of Two-Dimensional Echocardiograms. J Am Soc Echocardiogr, 1989; 2: 358-367.

16. Killip T 3rd, Kimball JT. Treatment of myocardial infarction in a coronary care unit. A two year experience with 250 patients. Am J Cardiol, 1967; 20: 457-464.

17. Levey AS, Coresh J, Balk E et al.; National Kidney Foundation. National Kidney Foundation practice guidelines for chronic kidney disease: Evaluation, classification, and stratification. Ann Intern Med, 2003; 139: 137-147. 
18. Mehran R, Nikolsky E. Contrast-induced nephropathy: definition, epidemiology, and patients at risk. Kidney Int Suppl, 2006; 100: S11-S15.

19. Schiele F, Legalery P, Didier K et al. Impact of renal dysfunction on 1-year mortality after acute myocardial infarction. Am Heart J, 2006; 151: 661-667.

20. Sarnak MJ, Levey AS, Schoolwerth AC et al.; American Heart Association Councils on Kidney in Cardiovascular Disease, High Blood Pressure Research, Clinical Cardiology, and Epidemiology and Prevention. Kidney disease as a risk factor for development of cardiovascular disease: A statement from the American Heart Association Councils on Kidney in Cardiovascular Disease, High Blood Pressure Research, Clinical Cardiology, and Epidemiology and Prevention. Hypertension, 2003; 42: 1050-1065.

21. Shlipak MG, Heidenreich PA, Noguchi H, Chertow GM, Browner WS, McClellan MB. Association of renal insufficiency with treatment and outcomes after myocardial infarction in elderly patients. Ann Intern Med, 2002; 137: 555-562.

22. Mann JF, Gerstein HC, Pogue J, Bosch J, Yusuf S. Renal insufficiency as a predictor of cardiovascular outcomes and the impact of ramipril: The HOPE randomized trial. Ann Intern Med, 2001; 134: 629-636.
23. Januzzi JL, Cannon CP, DiBattiste PM, Murphy S, Weintraub W, Braunwald E; TACTICS-TIMI 18 Investigators. Effects of renal insufficiency on early invasive management in patients with acute coronary syndromes (The TACTICS-TIMI 18 Trial). Am J Cardiol, 2002; 90: 1246-1249.

24. Januzzi JL, Cannon CP, Theroux P, Boden WE. Optimizing glycoprotein IIb/IIIa receptor antagonist use for the non-ST-segment elevation acute coronary syndromes: Risk stratification and therapeutic intervention. Am Heart J, 2003; 146: 764-774.

25. Rihal CS, Textor SC, Grill DE et al. Incidence and prognostic importance of acute renal failure after percutaneous coronary intervention. Circulation, 2002; 105: 2259-2264.

26. Schweiger MJ, Chambers CE, Davidson CJ et al. Prevention of contrast induced nephropathy: recommendations for the high risk patient undergoing cardiovascular procedures. Catheter Cardiovasc Interv, 2007; 69: 135-140.

27. McCullough PA, Wolyn R, Rocher LL, Levin RN, O'Neill WW. Acute renal failure after coronary intervention: incidence, risk factors, and relationship to mortality. Am J Med, 1997; 103: 368-375. 